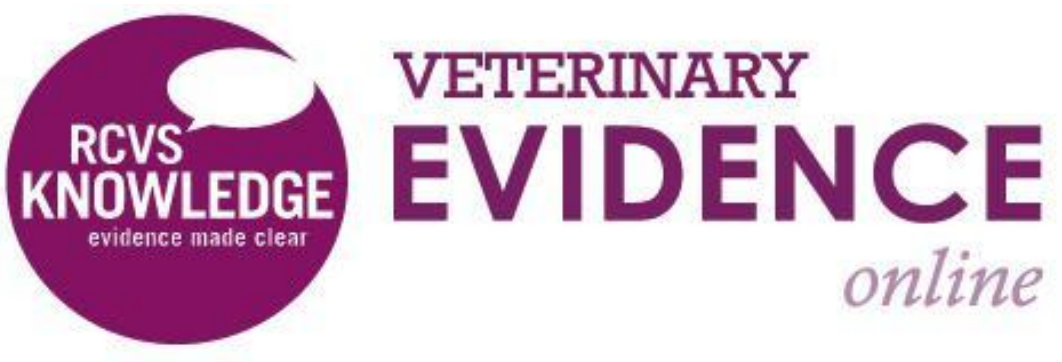

\title{
Does Adipose-Derived Stromal Cell Adjuvant Therapy for Fragmented Medial Coronoid Process in Dogs Influence Outcome? A Pilot Project
}

Kristina M. Kiefer DVM, PhD, CCRP, DACVSMR ${ }^{1^{*}}$

Katja Lin DVM, DACVS ${ }^{2}$

Noel Fitzpatrick Duniv MVB, CertVR, DSAO, ACVSMR, MRCVS ${ }^{3}$

Elizabeth Pluhar DVM, PhD, DACVS ${ }^{1}$

Michael G. Conzemius DVM, PhD, DACVS ${ }^{1}$

\footnotetext{
${ }^{1}$ University of Minnesota, College of Veterinary Medicine, 1365 Gortner Ave, St Paul, MN 55108, United States

${ }^{2}$ Las Vegas Veterinary Specialty Center, 650 W Tropicana Ave B-107, Las Vegas, NV 89147, United States

${ }^{3}$ Fitzpatrick Referrals, Halfway Lane, Eashing, Godalming, GU7 2QQ, United Kingdom

* Corresponding Author (kief0048@umn.edu)
}

ISSN: 2396-9776

Published: 9 Nov 2016

in: Vol 1, Issue 4

DOI: http://dx.doi.org/10.18849/ve.v1i4.45

Reviewed by: Adam Swallow (BVSc MRCVS) 
Objective: The primary objective of this study was to identify adverse events associated with multiple intraarticular injections of adipose stromal cell (ASC) therapy and secondarily to objectively assess the therapeutic effect of ASC therapy for treatment of fragmented medial coronoid process (FMCP) in dogs when used as an adjuvant to standard of care (SOC) treatment.

Background: Preliminary trials assessing autologous ASC therapy to treat osteoarthritis indicate a positive impact on clinical signs, but assessment of donated, allogeneic ASC therapy is lacking.

Evidentiary value: This prospective, randomised, controlled trial in dogs $(n=30)$ provides objective evidence for clinical practitioners regarding ASC therapy in a naturally occurring osteoarthritic disease model.

Methods: Dogs diagnosed with FMCP and osteoarthritis were enrolled. All dogs had arthroscopic fragment removal and proximal ulnar osteotomy (PUO) and were assigned into three groups ( $n=10 /$ group): 1 ) control group with no further treatment beyond the PUO and fragment removal (SOC), 2) PUO + autologous ASCs and 3) PUO+ allogeneic ASCs. Each dog had force platform gait analysis, Canine Brief Pain Inventory (CBPI) questionnaires, and delayed gadolinium enhanced magnetic resonance imaging scores prior to and six months after therapeutic intervention.

Results: No serious adverse events were reported in any participant. 3/10 dogs in the control group, 3/10 autologous ASC group and 7/10 allogeneic ASC group participants were assessed as successful outcomes.

Conclusion: This study provides preliminary safety data for the use of intra-articular allogeneic ASC therapy to treat osteoarthritis, and justification for larger clinical studies.

Application: Clinical practitioners considering ASC therapy within their practice are provided with additional evidence of autologous ASC therapy for osteoarthritis. Researchers committed to developing and generating effective ASC therapies are provided with safety information for allogeneic ASC, as well as identified biases important for study design.

\section{INTRODUCTION}

An estimated 1 in 5 dogs in the United States suffer from osteoarthritis affecting elbows, knees and hips ${ }^{1}$. Once the disease process begins, it is a non-reversible, progressive disease. Because current therapeutics do not cure this disease, a wide variety of therapeutic options are available, all of which have limitations and variable efficacy. Since the outcome of this disease process significantly affects quality of life in many patients and concurrent disease and/or adverse events may limit the pharmaceutical options that can be used, alternative therapies that impact outcome have generated great interest. Fragmentation of the medial coronoid process (FMCP) in the elbow of the dog often results in lameness and progressive osteoarthritis (OA), even after surgical management ${ }^{2-5}$.

Adipose derived stromal cells (ASC) have garnered interest for the treatment of osteoarthritic conditions in the dog, and following a small number of publications, have become a widespread therapy offered to owners ${ }^{6-11}$. These studies reported patient improvement compared to placebo control groups based on visually assessed lameness scores and caregiver-reports on improved quality of life ${ }^{6,7}$. Other studies demonstrated an 
improvement in force plate analysis after administration of $\mathrm{ASCs}^{8-10}$. These studies assess small numbers of cases and various inconsistent cellular therapies. The ASC therapy may consist of a stromal vascular fraction (SVF), which includes all unspecified, nucleated cells extracted from an autologous adipose tissue sample ${ }^{6,7}$, culture expanded $\mathrm{ASCs}^{11}$, or culture expanded ASCs combined with platelet rich plasma (PRP) ${ }^{8-10}$. These factors make clinical decision making when evaluating the value of ASC therapy challenging.

SVF therapy provides a rapidly produced and safe product for therapy of patients, but is a heterogeneous product that is inconsistent from patient to patient. Utilising a cultured and characterised preparation of ASC provides a less heterogeneous product that can be defined and selected for a specific purpose. Allogeneic cultured ASCs generate a therapeutic option for patients that cannot tolerate an anesthetic episode and are an immediately accessible treatment, as opposed to waiting several weeks for autologous cell culture. One study assessed dogs treated with autologous cultured ASCs in combination with PRP and found improved limb function using force platform gait analysis, indicating that cultured cells may still have a positive effect as a therapeutic ${ }^{8-10}$. Allogeneic cultured ASCs may provide the same clinical advantages of autologous cultured cells, but are a donated cell source. Allogeneic ASCs have been reported to improve limb function in people with knee OA without increased adverse events when compared to a placebo treated group ${ }^{12}$.

ASC therapy in OA is expected to take advantage of the immunomodulatory capacity and trophic effects that mesenchymal stem cells (MSC) provide ${ }^{13-17}$. ASCs in particular have a capacity to immunomodulate their environment, making them attractive as a therapy for active inflammatory conditions ${ }^{6-8,10,13-16,18-22}$. In addition to reducing inflammation and inflammatory responses, ASCs have the capacity to recruit local host cells that can induce a response that may not otherwise be present ${ }^{16,20}$.

The capacity of ASCs to immunomodulate is significant enough that allogeneic ASCs are better tolerated than other grafts, being accepted and incorporated into the local environment. They have been widely investigated for other immune modulating disease processes, but neglected in osteoarthritis in canine medicine ${ }^{23-25}$.

The primary objective of this study was to identify adverse events associated with multiple intra-articular injections of ASC therapy and secondarily to objectively assess the therapeutic effect of ASC therapy for treatment of FMCP in dogs when used as an adjuvant to standard of care (SOC) treatment. Additional objectives were to identify potential study biases and collect baseline outcome data to assist in future study designs and determine if a larger, more definitive randomised clinical trial was justified. Finally, to the authors' knowledge, this is the first reported use of delayed gadolinium enhanced magnetic resonance imaging (dGEMRIC) in a clinical population of naturally occurring OA in canines to assess cartilage quality.

METHODS \& MATERIALS

\section{Case Selection}

Thirty client-owned dogs (greater than 8-months of age and 10-kg body weight) that presented to University of Minnesota, College of Veterinary Medicine or Fitzpatrick Referrals, Eashing, Gadalming, Surrey, UK, for evaluation of a forelimb lameness with identified elbow pain secondary to a FMCP (confirmed by MRI and surgery) were included in this study. Dogs could be of any breed, sexual status or age. Informed owner consent for participation in the study with owner agreement to follow postoperative care guidelines and recheck examinations was required for participation in the study. Dogs that had previous elbow surgery, intra-articular 
injectable drug therapy for arthritis within 30 days of treatment, other systemic illnesses, or had other, concurrent, related (elbow incongruency, osteochondritis dissecans) or unrelated orthopedic or neurologic disease processes were excluded. All study procedures were in accordance with a protocol approved by the University of Minnesota Institutional Animal Care and Use Committee (IACUC \# 1203A11421).

\section{Experimental Groups}

Treatment groups included SOC (arthroscopic fragment removal and proximal ulnar osteotomy (PUO), SOC with autologous SVF therapy, or SOC with allogeneic ASC therapy. All dogs that were to receive cell therapies were treated at the University site in an effort to standardise cell preparation techniques and limit cell viability issues that might be associated with parcel delivery. Surgeons participating in the study standardised surgical technique so dogs would receive similar care regardless of treatment site. Complete transection of the ulna for PUO was confirmed at the time of surgery and with radiographic assessment post-operatively. At the university site, the first 15 enrolled dogs were randomly assigned to SOC or SOC+SVF therapy and the final 10 dogs were assigned to SOC+allogeneic ASC (complication with cell availability after the onset of the study delayed availability of this treatment group).

\section{Pre and Post-operative Care}

Anesthetic protocols were individually selected and designed by staff board certified anesthesiologists at the University of Minnesota. Post-operative management included hydromorphone ${ }^{\mathrm{a}} 0.05 \mathrm{mg} / \mathrm{kg}$ intramuscularly every six hours overnight, and beginning the following morning, oral tramadol ${ }^{b} 2-4 \mathrm{mg} / \mathrm{kg}$ every eight hours for ten days and a non-steroidal anti-inflammatory of the surgeon's choice administered per manufacturer's directions for ten days.

\section{Isolation of Adipose-derived Stromal Vascular Fraction}

Immediately following elbow surgery, dogs assigned to SOC with autologous SVF therapy had a ventral midline abdominal incision of $4-8 \mathrm{~cm}$ in length made between the xiphoid process and the umbilicus. The falciform adipose tissue was identified, exteriorised, and ligated with a circumferential 2-0 PDS suture prior to transection. The falciform tissue, approximately 30 grams, was placed into a sterile, covered container and transferred to the laboratory for processing. Falciform adipose tissue was harvested at the time of surgery and was processed according to previously reported protocols for isolation of the stromal vascular fraction overnight ${ }^{6,7,39}$. The nucleated fraction was used to determine cell number, and aliquoted into $5 \times 10^{\wedge} 6$ million nucleated cells/dose. SVF cells were cryopreserved in liquid nitrogen after the first treatment ${ }^{40}$. Isolation of Allogeneic Adipose-derived Stromal Cells

Falciform adipose tissue was harvested at the time of surgery from ten healthy dogs admitted to the University of Minnesota College of Veterinary Medicine for abdominal surgery unrelated to the study. Dogs with neoplastic or septic disease were excluded. Owner consent was obtained and all procedures were performed in accordance with the University of Minnesota Institutional Care and Use Committee (IACUC \# 1203A11421). Adipose tissue was processed according to previously reported protocols for isolation of the SVF ${ }^{6,7,41}$. The nucleated cell fraction was then placed into Keratinocyte $\mathrm{N}$ acetyl-L-cysteine supplemented (KNAC) medium consisting of modified MCDB153 medium (Keratinocyte-SFM) ${ }^{e}, \mathrm{MCDB} 153$ medium (Keratinocyte-SFM) ${ }^{e}, 2 \mathrm{mM}$ $\mathrm{N}$-acetyl-L-cysteine ${ }^{f}, 0.2 \mathrm{mM}$ L-ascorbic acid 2-phophate ${ }^{f} 0.09 \mathrm{mM}$ calcium and human recombinant epidermal growth factor $(5 \mathrm{ng} / \mathrm{mL})^{e}$, bovine pituitary extract $(50 \mathrm{ug} / \mathrm{mL})^{e}$, insulin $(5 \mathrm{ug} / \mathrm{mL})^{f}$, hydrocortisone ${ }^{f}(74 \mathrm{ng} / \mathrm{mL}), 5 \%$ 
fetal bovine serum ${ }^{g}$, and $1 \%$ antibiotic $^{h 15,3915,39}$. This protocol was chosen from amongst the treatments assessed in a previous study, to maximise tri-lineage differentiation, particularly chondrogenesis, and retention of $\mathrm{CD} 90, \mathrm{CD} 44$ and $\mathrm{MHCl}$ surface markers, as well as minimize pro-inflammatory mediators ${ }^{41}$. Cells were expanded to provide multiple doses per cell line, and harvested for therapy at passage three. Cells were then aliquoted as $5 \times 10^{\wedge} 6$ cells per dose and cryopreserved in liquid nitrogen until use ${ }^{40}$.

\section{Intra-articular injections}

Dogs assigned to either cell treatment group were given $0.05 \mathrm{mg} / \mathrm{kg}$ hydromorphone ${ }^{\mathrm{a}}$ and either $0.125 \mathrm{mg} / \mathrm{kg}$ diazepam $^{c}$ or $0.002-0.004 \mathrm{mg} / \mathrm{kg}$ dexmedetomidine hydrochloride ${ }^{\mathrm{d}}$ intravenously the day after surgery, prior to cell administration. All cell preparations were rinsed three times with sterile saline to remove medium or digestive agents from the preparation prior to administration. A preparation of either $5 \times 10^{\wedge} 6$ SVF nucleated cells or $5 \times 10^{\wedge} 6$ allogeneic ASCs constituted in $0.5 \mathrm{~mL}$ of sterile saline was injected intra-articularly into the operated elbow 24-hours after surgery. This protocol was repeated once, six weeks post-operatively. For the six-week injection and all allogeneic ASC injections, cells were thawed at room temperature prior to rinsing.

\section{Subjective Outcome Measures}

Owners completed a Canine Brief Pain Inventory (CBPI) questionnaire prior to group assignment, and six months post-operatively. The CBPI is a validated means of assessing owner opinion of outcome regarding pain and activity in dogs with clinical symptoms secondary to osteoarthritis ${ }^{42,43}$.

Radiographs were made prior to admission to study to confirm disease diagnosis. Dogs had radiographs made immediately post-operatively, at six weeks and at six months post-operatively. Each follow-up radiograph was evaluated for healing at the site of PUO. Osteotomy sites were classified as healed (site was bridged with osteosynthesis with little to no evidence of osteotomy site), not healed with osteogenic activity present, or not healed with no osteogenic activity.

\section{Objective Outcome Measures}

Force platform gait analysis was used to evaluate patient ground reaction forces (GRF), peak vertical force (PVF; the maximal force applied by the foot in weight bearing, perpendicular the platform) and vertical impulse (VI; a calculation of the force and time a limb is weight bearing ${ }^{44-46}$. Each dog was assessed prior to surgical intervention, and six months post-operatively. Data was collected by acquiring five valid trials for each forelimb at a walk on a force platform ${ }^{i}$ measuring $0.5 \mathrm{~m}^{2}$ in the center of a 10 meter runway. Velocity and acceleration were measured with the aid of five photoelectric cells coupled with a triggered timing mechanism and mounted 1 meter apart. A velocity range between $1-1.3 \mathrm{~m} / \mathrm{s}$, and an acceleration range of -0.5 to 0.5 $\mathrm{m} / \mathrm{s}^{2}$ was used to consider a trial valid. Data was collected at $1000 \mathrm{~Hz}$, and stored on a personal computer with software $e^{j}$ designed to record values velocity, acceleration, peak vertical force (PVF) and peak vertical impulse (VI). To be considered valid the entire foot had to make contact with the platform, without striking an edge, and the ipsilateral hind limb needed to follow with the same criteria. All dogs were weighed prior to data collection and trials were normalised to patient body weight.

The severity of OA was measured using delayed gadolinium enhanced magnetic resonance imaging (MRI) of cartilage (dGEMRIC) scores as previously described, utilising the methods and protocols (MRI sequences, coils, landmarks and post-processing techniques) published ${ }^{30}$. dGEMRIC was performed just prior to surgery and six months after surgery. After induction of anesthesia, each patient was administered gadopentetate dimeglumine ${ }^{k}(0.1 \mathrm{mmol} / \mathrm{kg})$ intravenously followed by ten minutes of passive range of motion of the affected 
elbow. MRI was performed as previously described, with a 3T system ${ }^{30}$. Scout images were evaluated, and two sagittal slices that included the medial coronoid process were selected for T1- weighted FSE inversion recovery sequences, to allow further processing with a data software program ${ }^{m}$, to generate a color map for each of the two images (dGEMRIC image) ${ }^{30}$. Each dGEMRIC image was evaluated and a ROI was drawn to include articular cartilage of the medial coronoid process, the corresponding articular cartilage of the humerus, and the intra-articular space in between ${ }^{30}$. The ROI was selected by the same individual for all cases and the individual was blinded to treatment group. Each image was evaluated three different times, with a new ROI identified for each assessment. The image intensity scores generated by the program from the ROI were averaged for the three images creating a single, average dGEMRIC score for each assessment period.

\section{Successful Outcome Definition}

Since the arithmetic mean is a less reliable description of group central tendency in studies with low power and in an effort to generate clinically applicable information, we chose to define criteria within each outcome measure that would classify the intervention as successful: Improved owner survey, near normal limb, and no progression of OA. For a case to have a successful outcome, a case had to meet all three criteria: 1) At the six month recheck there had to be a minimum of a ten percent improvement in CBPI survey scores for quality of life and lameness, 2) ground reaction forces (peak vertical force and vertical impulse) in the affected limb had to reach a minimum of eighty-five percent of normal forces measured in contralateral limbs at the six-month recheck ${ }^{47}, 3$ ) and there could not be a decrease in dGEMRIC score over the course of the study.

Adverse Events

Adverse events (AE) were defined as an observation in an enrolled animal that was unfavourable and unintended, and occurred after use of a cell treatment (SVF or allogeneic ASC) or surgical intervention at any time during the study period. Any abnormal clinical sign noted when the dog was in the hospital or reported by the owner was documented as an AE. Adverse events were classified as non-serious or serious and the relationship to the cell treatment was categorised as unknown, unlikely, possible, or probable.

\section{Statistical Analysis}

Statistical analysis was performed using SOFA Statistics software ${ }^{n}$. CBPI scores are reported as means of pain or function scores and were assessed using a Wilcoxon Signed Rank Sum test. GRF are reported as percentage of body weight $(100 * \mathrm{~N} / \mathrm{N})$ and values are expressed as means of the five collected trials at each assessment. dGEMRIC scores are reported as the mean of the all ROI generated for each outcome measure time point. Summary statistics are all reported as mean +/- standard deviation (SD). Differences between groups at the initial assessment, and at the 6-month recheck for GRF and dGEMRIC scores were evaluated using a one-way ANOVA test. Differences between initial exam and 6-month recheck for GRF and dGEMRIC scores were evaluated using a paired t-test. Differences in frequency of a successful outcome were tested using a Fisher's Exact test. Values of $p \leq 0.05$ were considered statistically significant.

\section{RESULTS}

Thirty dogs ( $n=10 /$ group) met the inclusion criteria and completed the study. Body weights ranged from 12.5 $\mathrm{kg}$ to $64 \mathrm{~kg}$, (31.82 kg+/-9.12). There was no significant difference between treatment groups with respect to 
body weight throughout the study. Patient age ranged from 0.66 years to 10 years (2.47yearst/-2.43). The allogeneic ASC treated group was significantly older (4.43years $+/-3.18, p=0.03$ ) than any of the other treatment groups, while no difference in age existed between the remaining two groups.

\section{Subjective Outcome Measures}

No significant difference was found between groups for pain and function scores on the CPBI scores at the initial or 6 month assessments. All groups improved pain and function scores at the 6 month assessment compared to the initial assessment (Figures 1 and 2). There was a significant decrease between initial pain score and 6 month recheck pain score in the SOC $(5.17+/-7.21, p<0.01)$ and autologous $(1.8+/-2.44, p<0.02)$, but not allogeneic $(5.87+/-4.23, p=0.06)$. There was a significant improvement between initial and 6 month function scores in function scores in the SOC $(3.7+/-7.6019, p<0.01)$ and autologous $(3.0+/-4.05518, p<0.01)$, but not allogeneic $(7.0+/-8.56, p=0.06)$. Dogs that had improvement in overall scores had an average improvement of $90.4 \%$ (range: $47.2-100 \%$ ).

All dogs had radiographic evidence of osteosynthesis at the PUO at 6-week recheck. 28/30 dogs were considered healed at the 6 week assessment. Of the two remaining dogs, one was considered healed at the 6 month recheck, and the final one was considered healed at a one-year recheck. Subjectively, all dogs had some shifting of the proximal ulna relative to the distal ulna on recheck radiographs, as compared to the postoperative radiographs.

\section{Objective Outcome Measures}

There were no significant differences in ground reaction forces (GRFs) between any of the groups at the initial assessment (PVF $p>0.06 ; \mathrm{VI} p>0.09$ for all groups) and at the 6-month assessment (PVF $p>0.26 ; \mathrm{VI} p>0.37$ ). (Figures 3 and 4) All groups had increased GRFs between initial and 6-month recheck. There was no significant change in VI in any of the groups ( $p>0.06$ ), but a statistically significant improvement was found in PVF of the SVF group ( $p=0.006$ and $p=0.002$, respectively).

There was no significant difference in dGEMRIC scores amongst groups at initial assessment (SOC 306.93+/62.14, SVF 337.43+/-39.19, AllSC 358.7+/-27.56, $p>0.05)$, or at the 6-month assessment $(359.15+/-53.88$, $329.37+/-50.49,365.5+/-60.83, p>0.05)$. A significant difference was identified between the initial score and the 6-month score in the SOC group, with the average 6-month score being higher. (Figure 5)

\section{Defining Success}

Using our defined criteria, thirty percent of cases (3 of 10) treated with proximal ulnar osteotomy (PUO) alone or autologous stromal vascular fraction (SVF) had successful outcomes. Seventy percent of cases (7 of 10) treated with allogeneic ASC met criteria to be considered successful. This difference was not statistically significant $(p=0.18)$.

\section{Adverse Events}

One dog in each treatment group (SOC, PUO + autologous ASC and PUO + allogeneic ASC) had a seroma develop at the surgical site of the PUO, reported or identified between 7-42 days after surgery. One dog (SOC+ allogeneic ASC) developed a swelling at the PUO site and was mildly febrile $\left(39.3^{\circ} \mathrm{C}\right) 4$ days after surgery. It was 
treated presumptively as an infection with $12.4 \mathrm{mg} / \mathrm{kg}$ Clavamox ${ }^{\circ}$ and resolved without further intervention. One dog (SOC+SVF) reportedly slipped, developed a worsening in lameness and was found to have a fissure at the PUO site 9 days after surgery. Lameness resolved with restricted activity. One dog (SOC) had redness and swelling at the surgical site 8 days post-operatively that resolved with $3.7 \mathrm{mg} / \mathrm{kg}$ firocoxib ${ }^{p}$. One dog (SOC+ allogeneic ASC) was found to be painful on palpation of the PUO surgical site at 42 days post-operatively, and was determined to have delayed healing of the osteotomy site. Radiographic healing was determined to be present at the 6-month recheck. Two dogs (SOC+SVF, SOC+ allogeneic ASC) were reported to have diarrhea. One of those dogs (SOC + allogeneic ASC) developed it two days prior to the 42 day recheck, while the other (SOC+ SVF) developed it within 24 hours of the second injection (39 days). The first resolved with $13 \mathrm{mg} / \mathrm{kg}$ metronidazole ${ }^{q}$, and the second was self limiting, resolving within 24 hours. No local swelling or erythematous reactions were reported by owners of dogs who received cellular therapy. All adverse events were considered non-serious.

\section{DISCUSSION}

To date, there are no published studies evaluating the use of allogeneic ASC to treat FMCP or OA in dogs, in spite of the many advantages they may provide over SVF. This pilot clinical study generates initial safety and efficacy data. To provide consistency in our assessments (and since the number of dogs/groups were small), we elected to investigate a single disease process, fragmented coronoid process (FMCP), which inevitably results in osteoarthritis of the canine elbow ${ }^{26}$. While this disease model involved surgery, the safety of use for multiple allogeneic intra-articular injections can be applied to any OA patient. The mean age of the dogs enrolled in the study is anticipated, given the disease process ${ }^{26-28}$. The allogeneic ASC group average age was significantly older than the other groups, and in particular included the two oldest dogs enrolled, at 8 and 10 years at time of enrollment. These two cases likely skewed the mean age of this group relative to the other three groups. This may also have skewed the outcome of this group, as the duration of the disease process may increase the severity of $O A$ and limit the ability of a patient to improve ${ }^{26-28}$. Identifying a correlation between severity of $O A$, body weight and age in regards to outcome would be useful information but was not assessed in this study due to the small numbers. Severity of preexisting OA may be an unidentified bias that influences response to therapy.

All dogs had improved CBPI scores from initial assessment to six-month rechecks, which could be explained by true or perceived improvement. Although owners could not choose group assignment, they were not blinded to the group they were assigned to, as it was apparent which groups had an abdominal incision. We did not feel it was ethically appropriate to perform surgical incisions in all dogs for the sole purpose of blinding the owners. Likewise, dogs receiving an ASC injection were sedated at the second injection, whereas the SOC was not, making it apparent to owners which group they had been assigned to. This is important to note, as the care-giver placebo effect can be quite potent ${ }^{29}$. The SOC + allogeneic ASC group had improvement of function based on scores, but pain scores at the 6-month recheck were higher compared to the SOC and SOC + SVF group. This group started at a higher pain score compared to the other two groups, and comparatively had a similar degree of improvement. Finally, owners were informed that allogeneic ASC treatment was experimental in nature, so this may have influenced owner responses.

Radiographs were assessed in each group six weeks after surgery to ensure progression to healing. The only noted radiographic problems with PUO were delayed healing in the oldest dog, and fissure following a traumatic incident. However, radiographic union did not appear to dramatically influence limb function as this dog achieved the clinical success guidelines at the 6-month time point.

Mean PVF and VI improved after intervention regardless of treatment group assignment. Dogs in the SOC + 
SVF group had the only statistically significantly difference in GRFs. The GRFs of these groups were lower at initial assessment, which may have allowed for a greater degree of improvement, and thus statistical significance. It cannot be ruled out that initial GRFs are predictive of outcome regardless of therapy.

Progression of OA is difficult to assess in a clinical study. Use of validated MRI techniques (e.g. dGEMRIC, T2mapping) helps resolve this problem by providing an objective estimation of cartilage health ${ }^{30}$. dGEMRIC provides a measurement of glycosaminoglycan content and T2-mapping provides a measurement of water content in the region of interest (ROI); in this study we used dGEMRIC. Since it has been reported that osteophytosis progresses even after surgical treatment of a FMCP, one would expect a decreased dGEMRIC score over time ${ }^{2-5}$. Mean dGEMRIC score decreased over time only in the SOC+SVF group. This change was not statistically significant and is not likely clinically relevant since it has been reported that, for the canine elbow, a change in dGEMRIC needs to approach $30 \mathrm{~ms}$ before being considered a real change (Wucherer, 2012). Possible explanations for no change in dGEMRIC over time include that a follow-up time of 6-months was too short and OA would have progressed if follow-up time were longer, or that glycosaminoglycan content in the medial compartment does not decrease short-term following the interventions studied even though subjective scoring of radiographic osteophytosis progresses. Dogs in the SOC group did have an increased dGEMRIC score. This could be explained by the changed pathomechanics in the elbow following the PUO ${ }^{31} 32$.

The dGEMRIC procedure had good inter- and intra-observational consistency when evaluated in normal dog elbows. ${ }^{30}$ While it has not been applied to abnormal dog elbows in a clinical situation prior to this study, it has been used to follow the progression of OA in people ${ }^{33}$. Since dogs with dysplastic elbows can have distorted anatomy (e.g. blunted coronoid processes, displaced coronoid) and that anatomy changes following coronoidectomy, it is possible that there is increased variability in the outcome measure in clinical patients because of inconsistent slice selection. However, throughout this study we noticed that the medial coronoid process was not particularly wide; and there were few choices where two slices (3-mm apart) could actually be selected during the MRI scan. Likewise, even if slice selection is consistent, coronoidectomy changes the anatomy that is evaluated and arguably increases average glycosaminoglycan in the ROI. For example, the region of the FMCP likely has the greatest amount of diseased cartilage. After this diseased region is removed the remaining components of the medial compartment would have a higher average glycosaminoglycan content. If these factors are valid, one could argue that dGEMRIC shortly after surgery should have been compared to the 6-month follow-up; that comparison may provide for a more accurate assessment of the response of the cartilage to the intervention over time.

Standard of care therapy used in this study has conflicting reports regarding the efficacy it may provide ${ }^{27,34-36}$. We may have found greater improvement between groups if a group that received no surgical intervention was included. However, since the objective of this study was to investigate any additional risks or benefits of adding biologic therapy to $\mathrm{SOC}$ we felt it more important to have SOC consistent across groups.

All adverse events, save one report of diarrhea (SOC +SVF), were considered unrelated to cellular therapy. The episode of diarrhea cannot be clearly attributed to the SVF injection versus the sedation drugs necessary to give the injection. There were no reactions to injections that would suggest a severe immune response. This provides no indication that even repeat intra-articular treatment with allogeneic ASCs add risk to the patient. Certainly, a systemically administered preparation (i.e. intravenous) could result in a different outcome.

One major limitation of this study is the small numbers of dogs in each group. In this pilot project, statistical differences, or lack thereof, need to be cautiously interpreted because 1) they are subject to Type I (incorrect rejection of null hypothesis) and Type II (incorrectly retaining null hypothesis) error and, 2) they are conflicting. For example, the SOC had the greatest improvement in dGEMRIC score, the SOC+SVF had the greatest improvement in GRFs, and the SOC+Allogeneic ASCs had the most dogs with a successful outcome. As expected in a study with only ten dogs/group it is difficult to draw a strong conclusion regarding a treatment by group effect. However, FMCP is a complicated disease and not fully understood ${ }^{26,27,37,38}$. 
We were able to identify study biases that will allow for improved study designs in the future. In this study population the greatest improvement in limb function came from the dogs with the greatest lameness. This is not surprising since they have the most room for improvement. The opposite may be true for the severity of $\mathrm{OA}$; dogs with severe OA at the onset may be limited in how much they can improve. Future studies should consider stratifying groups according to initial outcome measures to alleviate some of the bias that appeared within our groups. Finally, it is possible that the frequency of assessments and duration of follow-up should be different than the 6-months used in this study. It may be argued that the therapeutic benefit of ASCs could be shorter (needing more frequent observations) or much farther-reaching (needing longer duration of followup).

We identified no major contra-indications to repeat intra-articular injections of autologous or allogeneic ASC therapy, and provided some evidence that there may be a therapeutic benefit. Thus, we failed to reject our hypothesis that all treatments are well tolerated and safe for use. This study provides preliminary safety data for the use of allogeneic ASC therapy, and justification for larger clinical studies with greater numbers of dogs, stratification of participants based on initial limb function and dGEMRIC scores, and more observation periods and longer follow-up. It further adds objective outcome measures demonstrating some improvement in clinical signs for patients receiving autologous SVF. It also demonstrates the possibility of using dGEMRIC in naturally occurring osteoarthritis in dogs to assess OA progression.

\section{FOOTNOTES}

c Orion Corporation, NY, NY, USA

$d$ Hospira, IL, USA

$e$ Gibco, Life Technologies, Grand Island, New York, USA

$f$ Sigma-Aldrich, St. Louis, MO, USA

$g$ Hyclone, Thermo Fischer Scientific, Minneapolis, MN, USA

$h$ Mediatech Inc, Corning, NY, USA

i AMTI OR 6-5 force platform, Advanced Mechanical Technology, Watertown, Mass, USA

j Sharon Software Inc, Dewitt, MI, USA

$k$ Bayer Health Care Pharmaceuticals Inc, NJ, USA

I GE Sigma EXCITE Milwaukee, WI, USA

$m$ Matlab, MathWorks, Natick, Mass., USA

$n$ SOFA, v1.3.4, Paton- Simpson \& Associates Ltd, Auckland, New Zealand

$o$ Zoetis, Florham Park, NJ, USA

$p$ Merial, Duluth, GA, USA

$q$ Heritage Pharmaceuticals, Eatontown, NJ, USA

CONFLICT OF INTEREST

The authors declare no conflicts of interest. 
1. Johnston, S.A. Osteoarthritis. Joint anatomy, physiology, and pathobiology. Vet Clin North Am Small Anim Pract. 1997;27(4):699-723. http://www.ncbi.nlm.nih.gov/pubmed/9243777. Accessed September 5, 2016.

2. Evans, R.B. Gordon-Evans, and WJ. Conzemius, M.G. (2008) Comparison of Three Methods for the Management of Fragmented Medial Coronoid Process in the Dog. Veterinary and Comparative Orthopaedics and Traumatology, 21 (2), pp. 106-109. http://dx.doi.org/10.3415/VCOT-07-04-0031

3. Ness, M.G. (1998) Treatment of fragmented coronoid process in young dogs by proximal ulnar osteotomy. Journal of Small Animal Practice, 39 (1), pp. 15-18. http://dx.doi.org/10.1111/j.17485827.1998.tb03663.x

4. Snelling, S.R. and Lavelle, R.B. (2004) Radiographic Changes in Elbow Dysplasia Following Ulnar Osteotomy--A Case Report and Review of the Literature. Australian Veterinary Journal, 82 (5), pp. 278281. http://dx.doi.org/10.1111/j.1751-0813.2004.tb12703.x

5. Read, R.A. et al. (1990) Fragmentation of the Medial Coronoid Process of the Ulna in Dogs: A Study of 109 Cases. Journal of Small Animal Practice, 31 (7), pp. 330-334. http://dx.doi.org/10.1111/j.17485827.1990.tb00823.x

6. Black, L.L. et al. (2008) Effect of intraarticular injection of autologous adipose-derived mesenchymal stem and regenerative cells on clinical Signs of Chronic Osteoarthritis of the Elbow Joint in Dogs. Veterinary Therapeutics. 9 (3), pp. 192-200.

7. Black, L.L. et al. (2007) Effect of adipose-derived mesenchymal stem and regenerative cells on lameness in dogs with chronic osteoarthritis of the coxofemoral joints: a randomized, double-blinded, multicenter, controlled trial. Veterinary Therapeutics. 8 (4), pp. 272-284.

8. Guercio, A. et al. (2012) Production of canine mesenchymal stem cells from adipose tissue and their application in dogs with chronic osteoarthritis of the humeroradial joints. Cell Biology International, 36 (2), pp.189-194. http://dx.doi.org/10.1042/CBI20110304

9. Vilar, J.M. et al. (2013) Controlled, blinded force platform analysis of the effect of intraarticular injection of autologous adipose-derived mesenchymal stem cells associated to PRGF-Endoret in osteoarthritic dogs. BMC Veterinary Research, 9 (1), p. 131. http://dx.doi.org/10.1186/1746-6148-9$\underline{131}$

10. Vilar, J.M. et al. (2014) Assessment of the Effect of Intraarticular Injection of Autologous AdiposeDerived Mesenchymal Stem Cells in Osteoarthritic Dogs Using a Double Blinded Force Platform Analysis. BMC Veterinary Research, 10 (1), pp. 143 http://dx.doi.org/10.1186/1746-6148-10-143

11. Cuervo, B. et al. (2014) Hip Osteoarthritis in Dogs: A Randomized Study Using Mesenchymal Stem Cells from Adipose Tissue and Plasma Rich in Growth Factors. International Journal of Molecular Sciences, 15 (8), pp. 13437-13460 http://dx.doi.org/10.3390/ijms150813437

12. Vangsness, Jr, CTV. et al. (2014) Adult Human Mesenchymal Stem Cells Delivered via Intra-Articular Injection to the Knee Following Partial Medial Meniscectomy. The Journal of Bone and Joint SurgeryAmerican Volume, 96 (2), pp. 90-98. http://dx.doi.org/10.2106/JBJS.M.00058

13. Lee, J.M. et al. Comparison of Immunomodulatory Effects of Placenta Mesenchymal Stem Cells with Bone Marrow and Adipose Mesenchymal Stem Cells. International Immunopharmacology, 13 (2), pp. 219-224 http://dx.doi.org/10.1016/j.intimp.2012.03.024

14. Melief, S.M. et al. (2013) Adipose Tissue-Derived Multipotent Stromal Cells Have a Higher 
Immunomodulatory Capacity Than Their Bone Marrow-Derived Counterparts. Stem Cells Translational Medicine, 2 (6), pp. 455-463 http://dx.doi.org/10.5966/sctm.2012-0184

15. Kang, J.W. et al. (2008) Soluble factors-mediated immunomodulatory effects of canine adipose tissuederived mesenchymal stem cells. Stem Cells and Development, 17 (4), pp. 681693 http://dx.doi.org/10.1089/scd.2007.0153

16. Caplan, A.I. (2007) Adult Mesenchymal Stem Cells for Tissue Engineering Versus Regenerative Medicine. Journal of Cellular Physiology, 213 (2), pp. 341-347. http://dx.doi.org/10.1002/jcp.21200

17. Kuo, Y.R. et al. (2011) Modulation of Immune Response and T-Cell Regulation by Donor AdiposeDerived Stem Cells in a Rodent Hind-Limb Allotransplant Model. Plast Reconstr Surg. 128 (6), pp. 661e - 672e. http://dx.doi.org/10.1097/PRS.0b013e318230c60b

18. Frisbie, D.D. et al. (2009) Evaluation of adipose-derived stromal vascular fraction or bone marrowderived mesenchymal stem cells for treatment of osteoarthritis. Journal of Orthopaedic Research, 27 (12), pp.1675-1680 http://dx.doi.org/10.1002/jor.20933

19. Vilar, J.M. et al. (2013) Controlled, blinded force platform analysis of the effect of intraarticular injection of autologous adipose-derived mesenchymal stem cells associated to PRGF-Endoret in osteoarthritic dogs. BMC Veterinary Research, 9 (11), pp. 131 http://dx.doi.org/10.1186/1746-6148-9$\underline{131}$

20. Caplan, A.I. and Dennis, J.E. (2006) Mesenchymal stem cells as trophic mediators. Journal of Cellular Biochemistry, 98 (5), pp.1076-1084 http://dx.doi.org/10.1002/icb.20886

21. Wood, J.A. et al. (2012) Periocular and intra-articular injection of canine adipose-derived mesenchymal stem cells: an in vivo imaging and migration study. Journal of Ocular Pharmacology and Therapeutics, 28 (3), pp. 307-317.

22. Tyndall, A. and van Laar, J.M. (2010) Stem cells in the treatment of inflammatory arthritis. Best Practice \& Research Clinical Rheumatology, 24 (4), pp. 565-

574. http://dx.doi.org/10.1016/i.berh.2010.01.008

23. Cui, L. et al. (2007) Repair of cranial bone defects with adipose derived stem cells and coral scaffold in a canine model. Biomaterials, 28 (36), pp. 5477-

5486 http://dx.doi.org/10.1016/i.biomaterials.2007.08.042

24. Arinzeh, T. and Peter, S. (2003) Allogeneic mesenchymal stem cells regenerate bone in a critical-sized canine segmental defect. The Journal of Bone and Joint Surgery-American Volume, 85 (10), pp.19271935. http://dx.doi.org/10.2106/00004623-200310000-00010

25. Jung, D-I. et al. (2009) A comparison of autologous and allogenic bone marrow-derived mesenchymal stem cell transplantation in canine spinal cord injury. Journal of the Neurological Sciences, 285 (1-2), pp. 67-77 http://dx.doi.org/10.1016/i.jns.2009.05.027

26. Michelsen, J. (2013) Canine elbow dysplasia: Aetiopathogenesis and current treatment recommendations. The Veterinary Journal, 196 (1), pp. 12-

19 http://dx.doi.org/10.1016/i.tvil.2012.11.009

27. Fitzpatrick, N. (2009) Radiographic and arthroscopic findings in the elbow joints of 263 dogs with medial coronoid disease. Veterinary Surgery, 38 (2), pp. 213-223 http://dx.doi.org/10.1111/i.1532950X.2008.00489.x

28. Meyer-Lindenberg, A. et al. (2002) Prevalence of fragmented medial coronoid process of the ulna in lame adult dogs. Veterinary Record, 151 (8), pp. 230-234. http://dx.doi.org/10.1136/vr.151.8.230

29. Conzemius, M.G. and Evans, R.B. (2012) Caregiver placebo effect for dogs with lameness from 
osteoarthritis. Journal of the American Veterinary Medical Association, 241 (10), pp. 13141319. http://dx.doi.org/10.2460/javma.241.10.1314

30. Wucherer, K.L. et al. (2012) The use of delayed gadolinium enhanced magnetic resonance imaging of cartilage and t2 mapping to evaluate articular cartilage in the normal canine elbow. Veterinary Radiology \& Ultrasound, 53 (1), pp. 57-63. http://dx.doi.org/10.1111/i.1740-8261.2011.01867.x

31. Böttcher, P. et al. (2013) Estimation of Joint Incongruence in Dysplastic Canine Elbows Before and After Dynamic Proximal Ulnar Osteotomy. Veterinary Surgery, 42 (4) p.

371 http://dx.doi.org/10.1111/i.1532-950X.2013.01085.x

32. Fitzpatrick, N. et al. (2013) Bi-oblique dynamic proximal ulnar osteotomy in dogs: Reconstructed computed tomographic assessment of radioulnar congruence over 12 weeks. Veterinary Surgery, 42 (6), pp. 727-738 http://dx.doi.org/10.1111/j.1532-950X.2013.12014.x

33. Cunningham, T. et al. (2006) Delayed gadolinium-enhanced magnetic resonance imaging of cartilage to predict early failure of Bernese periacetabular osteotomy for hip dysplasia. The Journal of Bone and Joint Surgery (American), 88 (7), pp. 1540-1548. http://dx.doi.org/10.2106/JBJS.E.00572

34. Burton, N.J. et al. (2011) Conservative Versus Arthroscopic Management for Medial Coronoid Process Disease in Dogs: A Prospective Gait Evaluation. Veterinary Surgery, 40 (8), pp. 972-

980. http://dx.doi.org/10.1111/i.1532-950X.2011.00900.x

35. Evans, R.B. (2008) Comparison of three methods for the management of fragmented medial coronoid process in the dog. A systematic review and meta-analysis. Veterinary and Comparative Orthopaedics and Traumatology, 21, pp. 106-109 http://dx.doi.org/10.3415/VCOT-07-04-0031

36. Samoy, Y.C. et al. (2013) Arthroscopic treatment of fragmented coronoid process with severe elbow incongruity. Long-term follow-up in eight Bernese Mountain Dogs. Veterinary and Comparative Orthopaedics and Traumatology, 26 (1), pp. 27-33 http://dx.doi.org/10.3415/VCOT-11-06-0087

37. Samoy, Y.C. et al. (2012) Arthroscopic Findings in 32 Joints Affected by Severe Elbow Incongruity with Concomitant Fragmented Medial Coronoid Process. Veterinary Surgery 41, pp. 355-

361. http://dx.doi.org/10.1111/j.1532-950X.2012.00949.x

38. Vermote, K.A.G. et al. (2010) Elbow lameness in dogs of six years and older: arthroscopic and imaging findings of medial coronoid disease in 51 dogs. Veterinary and Comparative Orthopaedics and Traumatology, 23, pp. 43-50. http://dx.doi.org/10.3415/VCOT-09-03-0032

39. Kiefer, K.M. et al. (2014) The Influence of Culture Medium Type on Cellular Phenotype of Canine Adipose Derived Stem Cells. 3 (1), pp. 28-37. http://dx.doi.org/10.4236/ojrm.2014.31004

40. Gorin, N.C. (1978) Long-term preservation of bone marrow and stem cell pool in dogs. Blood, 51 (2), pp. 257-265.

41. Kiefer, K.M. et al. (2014) The Influence of Culture Medium Type on Cellular Phenotype of Canine Adipose Derived Stem Cells. Open Journal of Regenerative Medicine, 3 (1), pp. 28-

37. http://dx.doi.org/10.4236/ojrm.2014.31004

42. Brown, D.C. and Farrar, J.T. (2010) Development and psychometric testing of an instrument designed to measure chronic pain in dogs with osteoarthritis Dorothy. Journal of the American Veterinary MedicalAssociation, 68 (6), pp. 631-637.

43. Brown, D.C. et al. (2007) Development and psychometric testing of an instrument designed to measure chronic pain in dogs with osteoarthritis. American Journal of Veterinary Research, 68 (6), pp. 631-637.

44. Horstman, C.L. et al. (2004) Assessing the efficacy of perioperative oral carprofen after cranial cruciate 
surgery using noninvasive, objective pressure platform gait analysis. Veterinary Surgery, 33 (3), pp. 286-292. http://dx.doi.org/10.1111/j.1532-950x.2004.04042.x

45. Feldsien, J.D. et al. (2010) Serum cortisol concentration and force plate analysis in the assessment of pain associated with sodium urate-induced acute synovitis in dogs. American Journal of Veterinary Research. 71(8).

46. Moreau, M. et al. (2010) Influence of changes in body weight on peak vertical force in osteoarthritic dogs: A possible bias in study outcome. Veterinary Surgery, 39 (1), pp. 43-

47. http://dx.doi.org/10.1111/j.1532-950X.2009.00621.x

47. Rumph, P.F. et al. (1999) Interday variation in vertical ground reaction force in clinically normal Greyhounds at the trot. American Journal of Veterinary Research, 60 (6), pp. 679-683.

\section{TABLES \& CHARTS}

Figure 1: Comparison of pain scores between initial and follow-up assessments. Average score of canine brief pain inventory questionnaire for each treatment group's pain score is indicated at initial assessment (black), and at 6-month follow-up assessment (grey). The $x$ - axis is each treatment group, and the $y$-axis is the average score of owner assessment of patient pain calculated by answering four questions. ( $0=$ no pain, $10=$ extreme pain) The highest (worst pain) possible score would be forty. Treatments consisted of standard of care (SOC), autologous (Auto) or allogeneic (Allo) cell therapies. Letters above columns are to distinguish statistical significance. Columns labeled with the same letter indicate no statistical significance between scores. Columns that do not share a common alphabetic letter are $p<0.05$ and are considered statistically different.

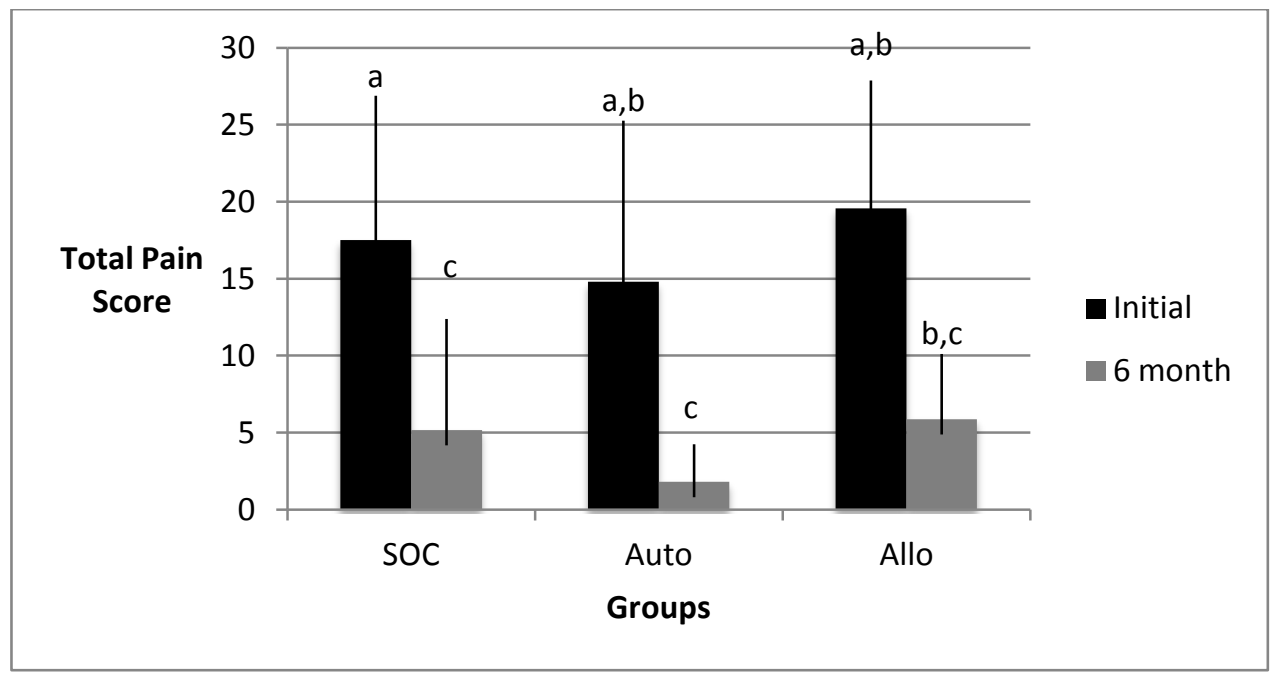


Figure 2: Comparison of function scores between initial and follow-up assessments. Average score of canine brief pain inventory questionnaire for each treatment group's function score at initial assessment (black), and at 6-month follow-up assessment (grey). The $x$ - axis is each treatment group, and the $y$-axis is the averaged score of owner assessment of patient function calculated by answering six questions. $(0=$ no interference, $10=$ completely interferes) The highest (worst) possible score would be sixty. Treatments consisted of standard of care (SOC), autologous (Auto) or allogeneic (Allo) cell therapies. Letters above columns are to distinguish statistical significance. Columns labeled with the same letter indicate no statistical significance between scores. Columns that do not share a common alphabetic letter are $p<0.05$ and are considered statistically different.

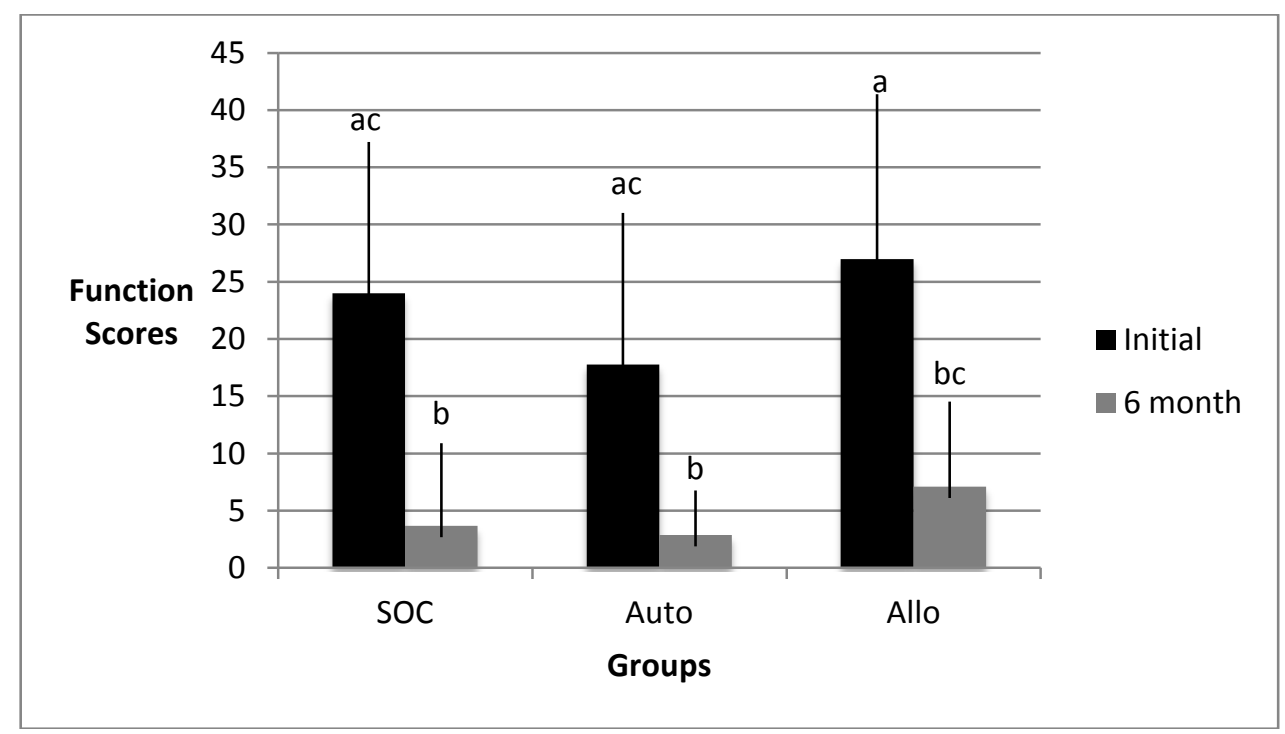

Figure 3: Comparison of peak vertical force between initial and follow-up assessments. Average peak vertical force, corrected for body weight, depicted on the $y$-axis, with each treatment group depicted on the $x$-axis. Initial assessment (black) and 6-month assessment (grey) are depicted side by side for each treatment group. Treatments consisted of standard of care (SOC), autologous (Auto) or allogeneic (Allo) cell therapies. Letters above columns are to distinguish statistical significance. Columns labeled with the same letter indicate no statistical significance between scores. Columns that do not share a common alphabetic letter are $p<0.05$ and are considered statistically different.

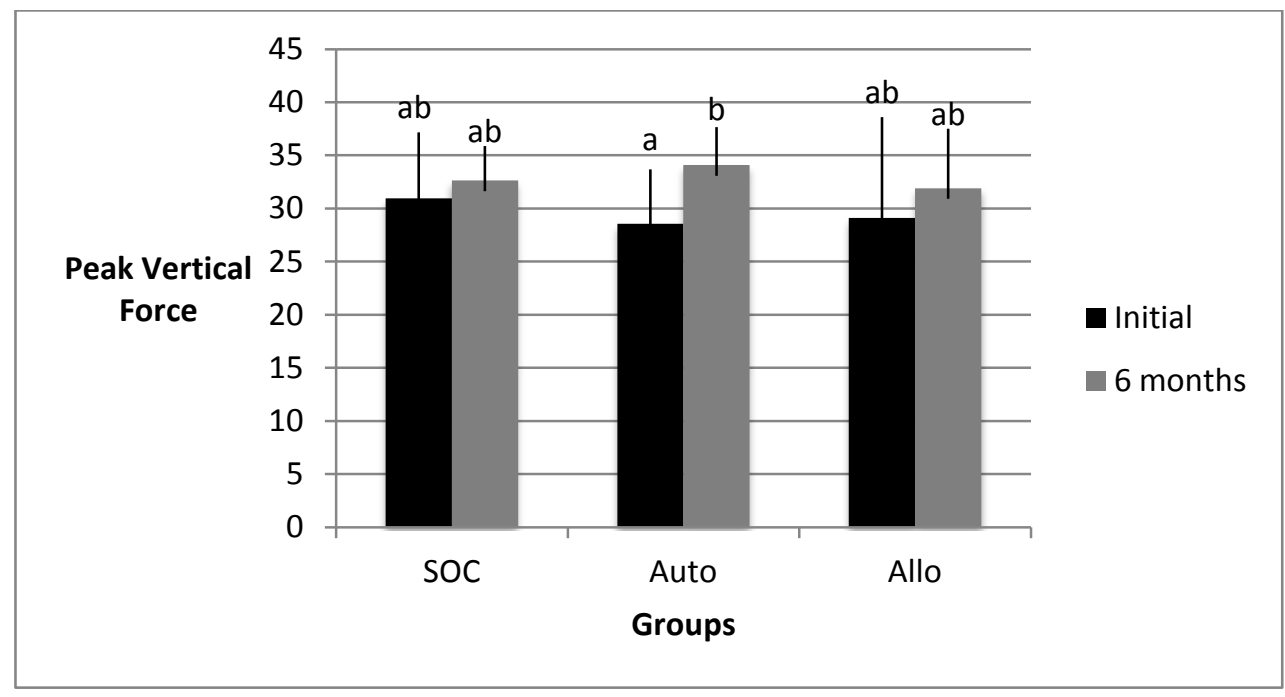


Figure 4: Comparison of peak vertical impulse between initial and follow-up assessments. Average peak vertical impulse, corrected for body weight, depicted on the $y$ - axis, with each treatment group depicted on the $x$-axis. Initial assessment (black) and 6-month assessment (grey) are depicted side by side for each treatment group. Treatments consisted of standard of care (SOC), autologous (Auto) or allogeneic (Allo) cell therapies. Letters above columns are to distinguish statistical significance. Columns labeled with the same letter indicate no statistical significance between scores. Columns that do not share a common alphabetic letter are $p<0.05$ and are considered statistically different.

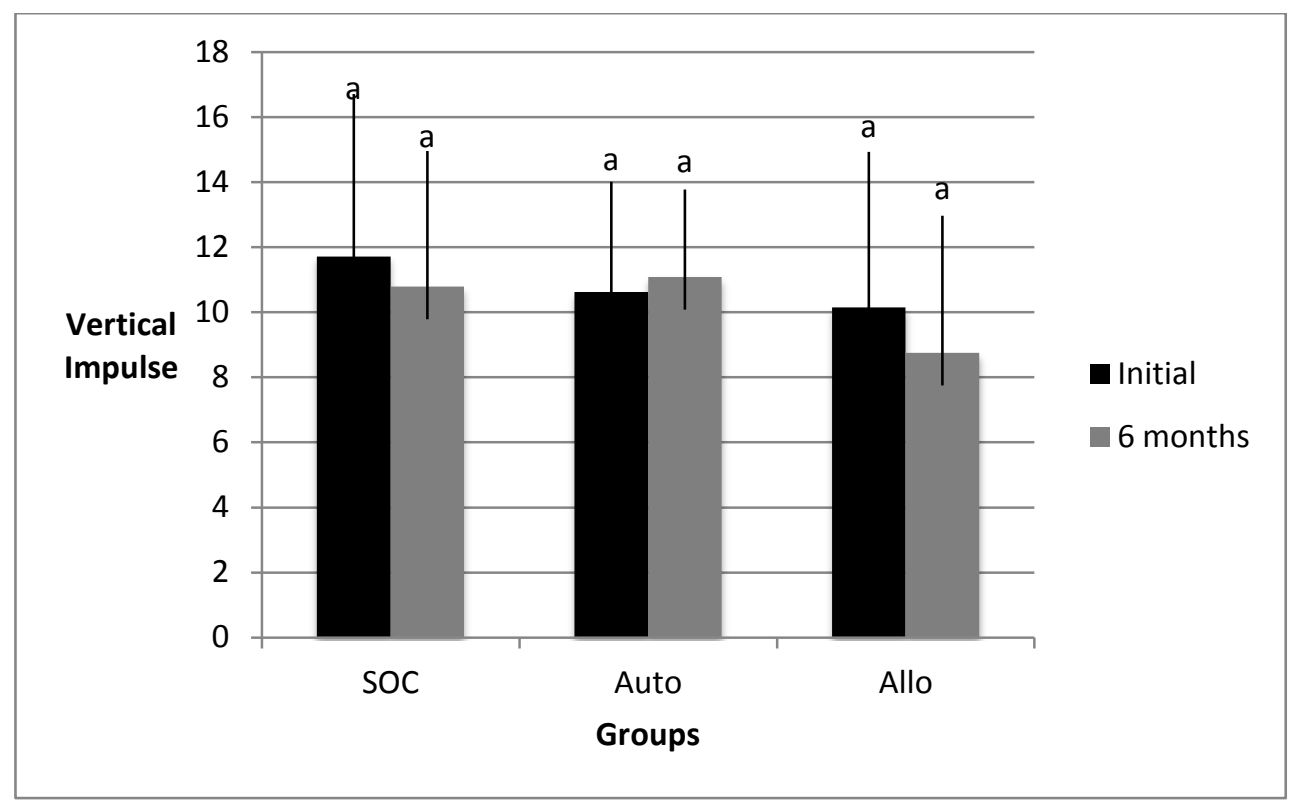

Figure 5: Comparison of dGEMRIC scores between initial and follow-up assessments. Averaged delayed gadolinium enhanced magnetic resonance imaging (MRI) of cartilage (dGEMRIC) score for each group depicted on the $y$-axis. Each treatment group is depicted on the $x$-axis at initial assessment (black) and 6 month recheck (grey) side by side. Treatments consisted of standard of care (SOC), autologous (Auto) or allogeneic (Allo) cell therapies. Letters above columns are to distinguish statistical significance. Columns labeled with the same letter indicate no statistical significance between scores. Columns that do not share a common alphabetic letter are $\mathrm{p}<0.05$ and are considered statistically different.

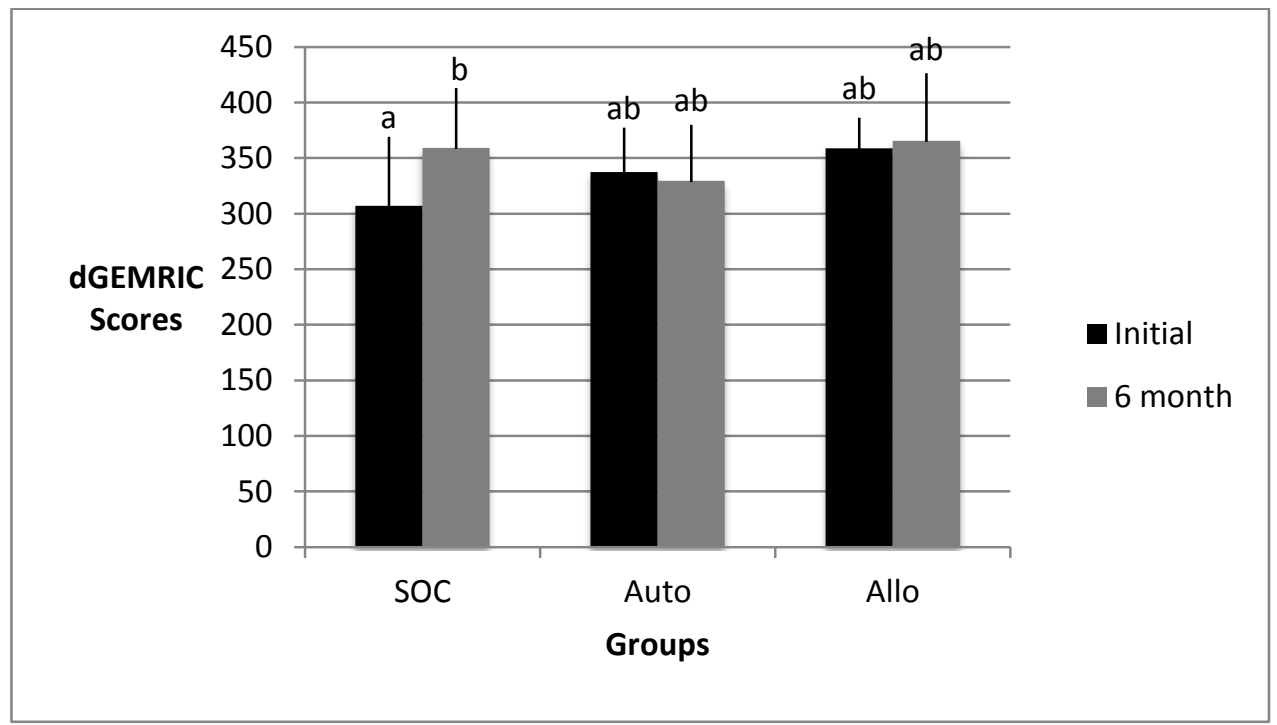




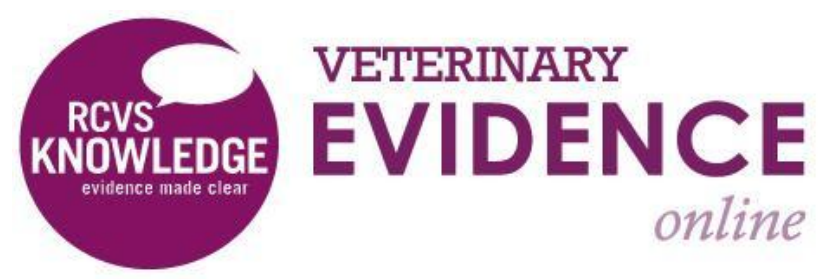

\section{Intellectual Property Rights}

Authors of Articles submitted to RCVS Knowledge for publication will retain copyright in their work, but will be required to grant to RCVS Knowledge an exclusive license of the rights of copyright in the materials including but not limited to the right to publish, re-publish, transmit, sell, distribute and otherwise use the materials in all languages and all media throughout the world, and to license or permit others to do so.

Authors will be required to complete a license for publication form, and will in return retain certain rights as detailed on the form.

Veterinary Evidence and EBVM Network are RCVS Knowledge initiatives. For more information please contact us at editor@veterinaryevidence.org.

RCVS Knowledge is the independent charity associated with the Royal College of Veterinary Surgeons (RCVS). Our ambition is to become a global intermediary for evidence based veterinary knowledge by providing access to information that is of immediate value to practicing veterinary professionals and directly contributes to evidence based clinical decision-making.

www.veterinaryevidence.org

RCVS Knowledge is a registered Charity No. 230886. Registered as a Company limited by guarantee in England and Wales No. 598443.

Registered Office:

Belgravia House

62-64 Horseferry Road

London SW1P 2AF

c) (7)

This work is licensed under a Creative Commons Attribution 4.0 International License. 